\title{
The Frequency of Lymph Node Metastases by Neck Level in p16 Positive Oropharyngeal Squamous Cell Carcinoma
}

\author{
Frederick Green ${ }^{1}$, Ali Moustafa ${ }^{1}$, Preetha Chengot ${ }^{1}$, Amit Prasai ${ }^{1}$, and Jim Moor ${ }^{2}$ \\ ${ }^{1}$ Leeds Teaching Hospitals NHS Trust \\ ${ }^{2}$ Leeds General Infirmary
}

June 28, 2020

\begin{abstract}
Objectives To determine the frequency by neck level of clinically known and occult lymph node metastasis in p16 positive oropharyngeal squamous cell carcinoma (p16+ve OPSCC). Design Retrospective study of 61 patients with p16+ve OPSCC whom had neck dissection and prior or simultaneous trans-oral surgery. Histopathology reports of neck dissection specimens were compared to preoperative clinical and radiological reports to determine the distribution of clinically known and occult nodal disease at each neck level and its concordance with preoperative findings. Positive and negative predictive values for pathological nodal disease ( $\mathrm{pN}+$ ve or $\mathrm{pN} 0)$ were assigned to clinically assessed node-positive ( $\mathrm{cN}+\mathrm{ve})$ or node-negative (cN0) status at each neck level. Setting Single tertiary referral centre in the UK Participants 61 patients with p16+ve OPSCC with no prior head and neck cancer treatment. Main outcome measures Clinical, radiological, and pathological findings of nodal disease in the neck. Results Two-hundred individual neck level specimens were analysed. Seventy-seven levels were considered $\mathrm{cN}+\mathrm{ve}$, of which $83.1 \%(64 / 77)$ were also pN+ve. One hundred and twenty-three levels were considered cN0 of which 13\% (16/123) were proven as $\mathrm{pN}+\mathrm{ve}$, demonstrating occult disease across various levels. This occult disease was identified in level II in 7 patients, level III in 6 patients and level IV in 3 patients, with no occult disease seen in cN0 level I or V. Conclusions These findings augment existing limited data on the distribution of occult nodes in OPSCC specific to p16+ve disease and reaffirm the rationale for dissecting at least levels II-IV in any cN0 neck.
\end{abstract}

\section{Abstract \\ Objectives}

To determine the frequency by neck level of clinically known and occult lymph node metastasis in p16 positive oropharyngeal squamous cell carcinoma (p16+ve OPSCC).

Design

Retrospective study of 61 patients with p16+ve OPSCC whom had neck dissection and prior or simultaneous trans-oral surgery. Histopathology reports of neck dissection specimens were compared to preoperative clinical and radiological reports to determine the distribution of clinically known and occult nodal disease at each neck level and its concordance with preoperative assessment. Positive and negative predictive values for pathological nodal disease ( $\mathrm{pN}+\mathrm{ve}$ or $\mathrm{pN} 0)$ were attributed to clinically assigned node-positive (cN+ve) or node-negative (cN0) status at each neck level.

\section{Setting}

Single tertiary referral centre in the UK

\section{Participants}

61 patients with p16+ve OPSCC with no prior head and neck cancer treatment. 


\section{Main outcome measures}

Clinical, radiological, and pathological findings of nodal disease in the neck.

Results

Two-hundred individual neck level specimens were analysed. Seventy-seven levels were considered cN+ve, of which $83.1 \%$ (64/77) were also pN+ve. One hundred and twenty-three levels were considered cN0 of which $13 \%(16 / 123)$ were proven as $\mathrm{pN}+\mathrm{ve}$, demonstrating occult disease across various levels. This occult disease was identified in level II in seven patients, level III in six patients and level IV in three patients, with no occult disease seen in $\mathrm{cNO}$ level I or V.

\section{Conclusions}

These findings augment existing limited data on the distribution of occult nodes in OPSCC specific to p16+ve disease and reaffirm the rationale for dissecting at least levels II-IV in any cN0 neck.

\section{Keywords}

Oropharyngeal cancer, Neck dissection, Lymphatic metastasis, Squamous cell carcinoma, Human papilloma virus, Tonsil Cancer, Cancer staging

\section{Key points}

- Patients with p16+ve OPSCC who are staged cN0 pre-treatment, and who undergo primary surgical treatment of the neck, are required to have a neck dissection including levels II, III and IV to avoid incomplete surgical management of occult nodal disease.

- If patients are staged $\mathrm{cN}+\mathrm{ve}$ pre-treatment, there is high positive predictive value for the presence of pathological nodal disease at the corresponding neck level.

- There is poor negative predictive value for the absence of nodal disease clinically at level II.

- The nodal distribution of HPV-specific disease is not significantly different to that found in previous studies that have not regarded HPV (or p16) status.

- We demonstrate a sensitivity of $93 \%$ and a specificity of $83 \%$ for cN0 staging in p16+ve OPSCC

\section{Background}

Seventy percent of oropharyngeal squamous cell carcinomas (OPSCC) are now associated with a positive p16 immunohistochemistry status (p16+ve OPSCC) in Europe and the USA, ${ }^{1,2}$ a number expected to increase. ${ }^{3}$ p16 positive immunohistochemistry is often used as a surrogate marker for the presence of the human papilloma virus (HPV). HPV-related OPSCC has doubled in incidence in the United Kingdom from 1990-2006 and again from 2006-2010. ${ }^{3}$

Compared to non-HPV associated OPSCC caused by exposure to associated carcinogens from tobacco and alcohol, patients affected by HPV associated disease represent a distinct population. Patients are of an average younger age, present at an earlier stage, and occupy a median higher socioeconomic class. ${ }^{4}$ Despite improved survival, quality-of-life indices remain low following treatment. ${ }^{5}$ It is therefore important to ensure that treatments, be they surgical or non-surgical, bring acceptably low recurrence risk whilst minimising loss of function.

Patients with p16+ve OPSCC typically present with cervical lymph node metastasis which may be asymptomatic, and in our institution are fully assessed clinically, radiologically and histologically with needle biopsy (or core biopsy if required) from the cervical lymph node/s and biopsy from the primary tumour site. Results are discussed at the multi-disciplinary team meeting and treatment is offered that may include primary surgery for patients whose tumours are deemed appropriate. This consists of trans-oral surgery to the primary tumour site (either using Trans-oral Laser Microsurgery (TLM), or Trans-Oral Robotic Surgery(TORS)) and selective neck dissection. ${ }^{3}$ Histopathological examination of neck dissection specimens 
then re-stages the resected tumour and nodal disease and identifies adverse features, which guide the decision on adjuvant non-surgical treatment.

The distribution of both clinically known and occult p16+ve OPSCC nodal disease in each neck level is not widely reported. Evaluation of this distribution could both reinforce the rationale for dissecting specific neck levels and evaluate the accuracy of preoperative clinical and radiological examination in establishing the presence and location of nodal disease.

\section{Materials and methods}

A retrospective review was conducted of patients who had undergone primary neck dissection (ND) for p16+ve OPSCC between 2015-2019 at our institution. NDs were performed by two surgeons (JWM and AP), and all patients underwent trans-oral surgery (TORS or TLM) of the primary tumour either simultaneously or after a delay of 1-2 weeks depending on logistical factors (availability of the surgical robot, for instance). Inclusion criteria included primary tumours with positive p16 immunohistochemistry (IHC) affecting the tonsil, base of tongue and soft palate, and patients for whom primary tumours were unidentifiable (for whom p16 positivity was established in IHC from lymph node metastasis). All patients with unidentifiable primary tumours underwent cross sectional imaging and PET/CT scan, bilateral tonsillectomy, and base of tongue mucosectomy to attempt to identify the primary site. Exclusion criteria included any patient who had received any form of previous head and neck cancer treatment, paediatric patients, non-oropharyngeal primary tumours, and p16 negative or 'p16 status unknown' tumour biology.

All neck dissections routinely include levels II-IV, with the ultimate extent of surgery being guided by pretreatment clinical and radiological staging. Excised nodal levels are resected en bloc and divided by the surgeon into separate specimens, each comprising a specific neck level. Each level is sent separately to histopathology fixed in formalin and processed according to an operating pathology protocol that safeguards specimen orientation, laboratory sampling, and the reporting of lymph nodes, extranodal extension, tumour margins and soft tissue deposits ${ }^{6}$. For the purpose of this analysis any patient who had separate specimens dissected from levels IIA and IIB were combined into a single 'Level II' to ensure consistency.

Assessments of clinical, radiological, and pathological reports were undertaken for each patient. Each neck level was assigned a status of clinically node-negative ( $\mathrm{cN} 0)$ or positive $(\mathrm{cN}+\mathrm{ve})$ and pathologically nodenegative ( $\mathrm{pN} 0)$ or positive $(\mathrm{pN}+\mathrm{ve})$. Occult nodal disease was defined as the pathological presence of metastatic nodal disease in the specimen with the absence of clinical or radiological disease specified at the corresponding neck level. Thereafter, neck node level of clinical and pathological disease statuses (cN0 or $\mathrm{cN}+\mathrm{ve}$, and $\mathrm{pN} 0$ or $\mathrm{pN}+\mathrm{ve}$ ) were recorded and compared.

Staging utilised the American Joint Committee on Cancer (AJCC) $8^{\text {th }}$ edition (TNM8) for p16+ve OPSCC. Clinical, radiological, and pathological reports of patients staged using the AJCC $7^{\text {th }}$ edition (TNM7) were reviewed to restage them according to the TNM8 criteria. The clinical staging pathway following clinical examination involves contrast-enhanced MRI of the neck and CT of the chest. In a minority of cases PET/CT or CT was used to stage the neck due to inability to undergo MRI.

Positive predictive values (PPV) and negative predictive values (NPV) for $\mathrm{cN}+$ ve status in each neck level were calculated with $95 \%$ confidence intervals (95\% CI) via the use of Microsoft Excel and are based on the chi-squared test for the ratios of two proportions. ${ }^{7,}{ }^{8}$ For parametric data, the unpaired Student's T test was used to assess the means between groups, with statistical significance assumed if $\mathrm{p}<0.05$.

\section{Results and analysis}

There were 61 patients included who underwent 63 NDs, of which 49 had a primary tonsillar tumours, 9 had primary tongue base tumours and 3 had unidentified primary tumours. Demographics, smoking history, clinical staging method and surgical details are shown in Table 1. A mean of 37.9 nodes were excised during each ND (SD 20.7) with cN0 necks yielding more nodes (mean 48.1) compared to cN+ve necks (mean 36.2, 
$\mathrm{p}=0.09)$, likely due to small sample sizes from cN0 necks. The mean number of positive nodes per ND was 3.3 (median 2, range 1-19).

Overall, one patient was downstaged on pathological nodal staging (from $\mathrm{cN} 1$ to pN0). Eleven of the sixtyone patients were upstaged, (seven from $\mathrm{cN} 1$ to $\mathrm{pN} 2$ and four from $\mathrm{cN} 0$ to $\mathrm{pN} 1$ ). Staging for the remaining forty-nine patients was unchanged.

\section{Overall distribution of lymph node metastases}

The frequency of LN metastases at each neck level is shown in Table 2. Overall, fifty-four of the sixty-three NDs were performed in $\mathrm{cN}+$ ve necks; of these, fifty-three were subsequently staged as $\mathrm{pN}+\mathrm{ve}$ and one as pN0. The remaining nine NDs were performed in cN0 necks. Of these four (44\%) were subsequently pN+ve, of which disease was invariably in Level II, and five remained pN0. This yielded an overall sensitivity of $93 \%$ and a specificity of $83 \%$ for $\mathrm{cN} 0$ staging in p16+ve OPSCC.

\section{Level-specific analysis}

Table 3 examines the frequency of nodal metastases in each neck level in specimens that were cN0 and $\mathrm{cN}+\mathrm{ve}$, respectively, at the corresponding level. 'Occult' nodes for each level were from here defined as those found on pathological staging in levels that had been $\mathrm{cN0}$ at the corresponding level. It therefore demonstrates the concordance of ND specimens with preoperative clinical and radiological examination.

Two hundred individual neck level specimens were analysed (see Table 2) from the sixty-one patients. Seventy-seven neck level specimens had been ascribed $\mathrm{cN}+$ ve status at the corresponding level, of which $83.1 \%(64 / 77)$ had pathological evidence of metastatic disease. One hundred and twenty-three neck level specimens were $\mathrm{cN0}$ in the corresponding level, of which occult disease was found in $13.0 \%(16 / 123)$. This occult disease was found in $58.3 \%$ (7/12) of cN0 level IIs, $13.3 \%(6 / 45)$ of cN0 level IIIs, $5.5 \%(3 / 61)$ of cN0 level IVs, and $0 / 5 \mathrm{cN0}$ level Vs. Overall, sixteen of sixty-one patients had pathological nodes in levels not established clinically. All four level I NDs were performed on necks $\mathrm{cN}+\mathrm{ve}$ for level I, of which three were $\mathrm{pN}+$ ve in level I; two of the seven level V NDs were performed on necks $\mathrm{cN}+$ ve for level $\mathrm{V}$, of which one was $\mathrm{pN}+$ ve in level $\mathrm{V}$.

Table 4 presents the negative predictive values (NPV) and positive predictive values (PPV) for cN0 and $\mathrm{cN}+$ ve status, respectively, for each level. With regard to the distribution of lymph node metastasis at adjacent neck levels, it was found that patients who were $\mathrm{cN}+$ ve at level II had a PPV of $30.9 \%$ (95\% CI $27.4 \%-34.6 \%$ ) for also being pN+ve at level III, and those who were $\mathrm{cN0}$ at level II had a NPV of $87.5 \%$ $(95 \%$ CI $48.1 \%-98.2 \%)$ for being pN0 at level III. Patients who were $\mathrm{cN}+$ ve at level III had a PPV of $11.1 \%$ (95\% CI 4.8\% - 23.6\%) for also being pN+ve in level IV, and a NPV of $97.8 \%$ (95\% CI $89.8 \%-99.6 \%$ ) for the absence of pathological level IV nodes (data not shown).

\section{Discussion}

This study examines the distribution of clinically known and occult nodal disease in neck dissection specimens of patients with p16+ve OPSCC who have undergone primary treatment with TORS/TOLM and ND. The data reaffirms previously reported data on the importance of including levels II, III and IV in ND for any $\mathrm{cN}+\mathrm{ve}$ p16+ve OPSCC, although the volume of previously published literature is low ${ }^{9-11}$. The frequency of occult node metastasis in patients staged overall as cN0 (four of nine NDs, 44\%) underlines the importance of sound oncological surgical technique when undertaking selective neck dissection in this cohort. There is no place for limiting the neck dissection to levels II and III; levels II, III and IV is the minimum neck dissection patients should be offered regardless of the absence of clinical disease in prior echelon levels.

Three other studies previously published have examined the nodal distribution specific to p16+ve disease $9,10,12$, with just one comparing the breakdown of clinically known and occult nodes ${ }^{10}$. Previous studies had examined the distribution pattern of known and occult OPSCC nodal disease irrespective of p16 status, ${ }^{4,} 13-17$ with HPV only relatively recently demarcated as a disease subtype with a specific natural history and 
transmission. ${ }^{3}$ A recent Zenga et al. study examined a cohort of $324 \mathrm{pN}+\mathrm{ve}$ p16+ve OPSCC patients; however this study did not assess the distribution of LNs by neck level. ${ }^{11}$

The data we report here is consistent with studies reporting the frequency of occult lymph node metastasis in equivalent patient groups in level II (24-80\%), level III (0-60\%), and level IV nodes $(0-27 \%) .4,10,15,17$ The reported frequency of all LN metastasis are $76-90 \%$ for level II, $22-50 \%$ for level III and $9-14 \%$ for level IV which are comparable with our data. $4,9,10,12,13,17$. The insight gained from this study is similar to Amsbaugh et al who compared nodal distribution frequency in p16+ve and p16-ve OPSCC, ${ }^{12}$ but which only examined the distribution of $\mathrm{cN}+\mathrm{ve}$ LNs. This found that the distribution of nodes in $\mathrm{p} 16+\mathrm{ve}$ disease was not significantly different to an historical Lindbergh study on overall nodal distributions in OPSCC conducted prior to establishment of the HPV aetiology. ${ }^{13}$ However, the Amsbaugh et al study had a $67 \%$ rate of smokers, introducing a confounding risk factor compared to our cohort with only $39 \%$ of patients reporting as current or significant smokers in the past.

We acknowledge that there is a weakness in our reporting of level IIA and IIB disease as this was only available for a small subset of patients (table 2), however with the limited data available we have shown that the presence of level IIB disease was always associated with level IIA disease. Generally, the senior authors' policy is to dissect level IIA and IIB as standard in this patient group, but these specimens have, for the majority, been sent as a single level II specimen without sub-division. A larger study found isolated IIB disease in $2.2 \%$ without level IIA disease, although none in cN0 necks. ${ }^{4}$ Recommendations in the United Kingdom remain that IIB can be left intact in T1-2 tumours if clinical disease in IIA is absent, ${ }^{3,4}$ with heightened suspicion in tonsillar tumours. ${ }^{4}$

As with this study, previous reports on occult nodes for NDs reported uncommon involvement of levels I and $\mathrm{V}$, with frequencies of $0-9 \%$ for level I and of $0-7 \%$ for level $\mathrm{V} .{ }^{4}, 10,14,{ }^{15}$ Dissection of level I is not considered routine and is advised in the presence of anterior tumour expansion into the oral cavity or in the presence of $\mathrm{cN}+$ ve level I lymph nodes. ${ }^{3}$

\section{Limitations}

In this cohort, only two patients had a bilateral ND, with both patients having both necks staged as $\mathrm{cN}+\mathrm{ve}$ (and therefore, no occult nodes found). Other studies have described frequencies of nodes in contralateral dissections as well as ipsilateral, although with insufficient frequencies of occult nodes to justify routine contralateral ND in all cases. ${ }^{4}, 10,12,13,17$ In patients who are managed with primary surgery to the contralateral neck, further analytical work is required to demonstrate the pattern of contralateral lymph node metastasis.

Lastly, despite the high negative predictive value of p16-ve protein expression for the absence of HPV, Royal College of Pathology advice recommends in-situ hybridisation of all p16+ve specimens to confirm HPV, which was not routinely available at our institution during the study period done. We estimate that $4 \%$ of patients excluded from the analysis on the basis of having p16-ve disease may therefore have been eligible for inclusion; exact data is, however, not known. ${ }^{18}$

\section{Conclusion}

We have demonstrated that level II, III and IV neck dissection is the minimum number of levels required to be dissected for all patients undergoing primary surgery for p16+ve OPSCC. Supra-selective ipsilateral neck dissection in the primary surgical setting cannot be recommended due to the potential risk of undertreatment of occult disease in level IV. We also demonstrate that the $\mathrm{cN} 0$ neck at level II has a low negative predictive value for the absence of clinical nodal disease at level II, although the clinical utility of this is low.

\section{References}

1. Mehanna H., Beech T., Nicholson T., El-Hariry I., McConkey C., Paleri V., et al. Prevalence of human papillomavirus in oropharyngeal and nonoropharyngeal head and neck cancer-systematic review and metaanalysis of trends by time and region. Head Neck. 2013;35(5):747-55. 
2. Näsman A., Attner P., Hammarstedt L., Du J., Eriksson M., Giraud G., et al. Incidence of human papillomavirus (HPV) positive tonsillar carcinoma in Stockholm, Sweden: an epidemic of viral-induced carcinoma? Int J Cancer. 2009;125(2):362-6.

3. Mehanna H., Evans M., Beasley M., Chatterjee S., Dilkes M., Homer J., et al. Oropharyngeal cancer: United Kingdom National Multidisciplinary Guidelines. J Laryngol Otol. 2016;130(S2):S90-s6.

4. Gross B.C., Olsen S.M., Lewis J.E., Kasperbauer J.L., Moore E.J., Olsen K.D., et al. Level IIB lymph node metastasis in oropharyngeal squamous cell carcinoma. Laryngoscope. 2013;123(11):2700-5.

5. Sharma A., Méndez E., Yueh B., Lohavanichbutr P., Houck J., Doody D.R., et al. Human papillomaviruspositive oral cavity and oropharyngeal cancer patients do not have better quality-of-life trajectories. Otolaryngol Head Neck Surg. 2012;146(5):739-45.

6. Standard Operating Procedure: Reporting of neck dissection specimens. The Leeds Teaching Hospitals NHS Trust 2020.

7. Newcombe R. Excel spreadsheet to calculate and display positive and negative predictive values as functions of prevalence 2016 [Available from: https://www.researchgate.net/publication/305073460_Excel_spreadsheet_to_calculate_and_display_positive_and_negative_predictive_values_as_functions_of_prevalence.

8. Miettinen O., Nurminen M. Comparative analysis of two rates. Stat Med. 1985;4(2):213-26.

9. Cannon R.B., Houlton J.J., Patel S., Raju S., Noble A., Futran N.D., et al. Patterns of cervical node positivity, regional failure rates, and fistula rates for HPV+ oropharyngeal squamous cell carcinoma treated with transoral robotic surgery (TORS). Oral Oncol. 2018;86:296-300.

10. Plonowska K.A., Strohl M.P., Wang S.J., Ha P.K., George J.R., Heaton C.M., et al. Human Papillomavirus-Associated Oropharyngeal Cancer: Patterns of Nodal Disease. Otolaryngol Head Neck Surg. 2019;160(3):502-9.

11. Zenga J., Jackson R.S., Graboyes E.M., Sinha P., Lindberg M., Martin E.J., et al. Oncologic outcomes of selective neck dissection in HPV-related oropharyngeal squamous cell carcinoma. Laryngoscope. 2017;127(3):623-30.

12. Amsbaugh M.J., Yusuf M., Cash E., Silverman C., Wilson E., Bumpous J., et al. Distribution of Cervical Lymph Node Metastases From Squamous Cell Carcinoma of the Oropharynx in the Era of Risk Stratification Using Human Papillomavirus and Smoking Status. Int J Radiat Oncol Biol Phys. 2016;96(2):349-53.

13. Lindberg R. Distribution of cervical lymph node metastases from squamous cell carcinoma of the upper respiratory and digestive tracts. Cancer. 1972;29(6):1446-9.

14. Candela F.C., Kothari K., Shah J.P. Patterns of cervical node metastases from squamous carcinoma of the oropharynx and hypopharynx. Head Neck. 1990;12(3):197-203.

15. Shah J.P. Patterns of cervical lymph node metastasis from squamous carcinomas of the upper aerodigestive tract. Am J Surg. 1990;160(4):405-9.

16. Robbins K.T., Ferlito A., Shah J.P., Hamoir M., Takes R.P., Strojan P., et al. The evolving role of selective neck dissection for head and neck squamous cell carcinoma. Eur Arch Otorhinolaryngol. 2013;270(4):1195202.

17. Lim Y.C., Koo B.S., Lee J.S., Lim J.Y., Choi E.C. Distributions of cervical lymph node metastases in oropharyngeal carcinoma: therapeutic implications for the N0 neck. Laryngoscope. 2006;116(7):1148-52.

18. Craig S.G., Anderson L.A., Schache A.G., Moran M., Graham L., Currie K., et al. Recommendations for determining HPV status in patients with oropharyngeal cancers under TNM8 guidelines: a two-tier approach. Br J Cancer. 2019;120(8):827-33. 
Table 1. Patient demographics and types of neck dissection. Value presented as number (\%) unless stated otherwise. All $p$

Number of patients

Age, mean (range) $(\mathrm{n}=61)$

$\operatorname{Sex}(\mathrm{n}=61)$

Smoking history $(\mathrm{n}=61)$

Imaging used for clinical staging $(\mathrm{n}=61)$

Neck dissections $(\mathrm{n}=63)$

Levels dissected $(\mathrm{n}=63)$

Table 2. Overall frequency of dissection of each level and identification of lymph node metastasis at that level

Neck Level Dissected

I

II ${ }^{\S}$

III

IV

$\mathrm{V}$

Total

${ }^{\S}$ Level II was subdivided into IIA and IIB in 26 of the 63 dissection specimens; LN metastasis was found in 24 of 26 IIA spe

Table 3. Comparison of clinical and histological staging at each neck level for both $\mathrm{cN} 0$ and $\mathrm{cN}+\mathrm{ve}$ patients in all neck dis Neck level

I

II

III

IV

V 


\begin{tabular}{|c|c|c|c|c|}
\hline $\begin{array}{l}\text { Table } 4 . \\
\text { Comparison of } \\
\text { the negative } \\
\text { predictive value } \\
\text { (NPV) for the } \\
\text { absence of clinical } \\
\text { disease (cN0 } \\
\text { status) in each } \\
\text { neck level and the } \\
\text { positive predictive } \\
\text { value (PPV) of } \\
\mathrm{cN}+\text { ve status in } \\
\text { each neck level }\end{array}$ & $\begin{array}{l}\text { Table } 4 . \\
\text { Comparison of } \\
\text { the negative } \\
\text { predictive value } \\
\text { (NPV) for the } \\
\text { absence of clinical } \\
\text { disease (cN0 } \\
\text { status) in each } \\
\text { neck level and the } \\
\text { positive predictive } \\
\text { value (PPV) of } \\
\text { cN+ve status in } \\
\text { each neck level }\end{array}$ & $\begin{array}{l}\text { Table } 4 . \\
\text { Comparison of } \\
\text { the negative } \\
\text { predictive value } \\
\text { (NPV) for the } \\
\text { absence of clinical } \\
\text { disease (cN0 } \\
\text { status) in each } \\
\text { neck level and the } \\
\text { positive predictive } \\
\text { value (PPV) of } \\
\text { cN+ve status in } \\
\text { each neck level }\end{array}$ & $\begin{array}{l}\text { Table } 4 . \\
\text { Comparison of } \\
\text { the negative } \\
\text { predictive value } \\
\text { (NPV) for the } \\
\text { absence of clinical } \\
\text { disease (cN0 } \\
\text { status) in each } \\
\text { neck level and the } \\
\text { positive predictive } \\
\text { value (PPV) of } \\
\mathrm{cN}+\text { ve status in } \\
\text { each neck level }\end{array}$ & $\begin{array}{l}\text { Table } 4 . \\
\text { Comparison of } \\
\text { the negative } \\
\text { predictive value } \\
\text { (NPV) for the } \\
\text { absence of clinical } \\
\text { disease (cN0 } \\
\text { status) in each } \\
\text { neck level and the } \\
\text { positive predictive } \\
\text { value (PPV) of } \\
\text { cN+ve status in } \\
\text { each neck level }\end{array}$ \\
\hline Neck level & NPV of cN0 (\%) & $95 \% \mathrm{CI}$ & $\begin{array}{l}\text { PPV of } c N+v e \\
(\%)\end{array}$ & $95 \% \mathrm{CI}$ \\
\hline I & 100 & na & 75.0 & $30.1-95.4$ \\
\hline II & 41.7 & $22.9-63.2$ & 94.1 & $86.7-97.5$ \\
\hline III & 86.7 & $77.0-92.7$ & 66.7 & $47.0-81.2$ \\
\hline IV & 95.1 & $94.9-95.3$ & $0^{\S}$ & na \\
\hline $\mathrm{V}$ & 100 & na & 50.0 & -87.5 \\
\hline$\S$ No cN+ve level & $\S$ No cN+ve level & $\S$ No cN+ve level & $\S$ No cN+ve level & $\S \mathrm{No} \mathrm{cN}+\mathrm{ve}$ level \\
\hline $\begin{array}{l}\text { IV ND specimens } \\
\text { returned } \mathrm{pN}+\mathrm{ve} \\
\text { disease in level }\end{array}$ & $\begin{array}{l}\text { IV ND specimens } \\
\text { returned pN+ve } \\
\text { disease in level }\end{array}$ & $\begin{array}{l}\text { IV ND specimens } \\
\text { returned pN+ve } \\
\text { disease in level }\end{array}$ & $\begin{array}{l}\text { IV ND specimens } \\
\text { returned } \mathrm{pN}+\mathrm{ve} \\
\text { disease in level }\end{array}$ & $\begin{array}{l}\text { IV ND specimens } \\
\text { returned pN+ve } \\
\text { disease in level }\end{array}$ \\
\hline IV; all level IV & IV; all level IV & IV; all level IV & IV; all level IV & IV; all level IV \\
\hline $\mathrm{pN}+$ ve disease & $\mathrm{pN}+$ ve disease & $\mathrm{pN}+\mathrm{ve}$ disease & $\mathrm{pN}+\mathrm{ve}$ disease & $\mathrm{pN}+$ ve disease \\
\hline was occult (see & was occult (see & was occult (see & was occult (see & was occult (see \\
\hline Table 3) & Table 3) & Table 3) & Table 3) & Table 3) \\
\hline
\end{tabular}

